\title{
Well-Being Reduces COVID-19 Anxiety: A Three-Wave Longitudinal Study in China
}

\author{
Zhuojun Wang $^{1} \cdot$ Shuyi Luo ${ }^{1}$. Jianjie Xu ${ }^{1} \cdot$ Yanwei Wang ${ }^{1} \cdot$ Hanqi Yun ${ }^{1} \cdot$ Zihao Zhao $^{1}$. \\ Haocheng Zhan ${ }^{1} \cdot$ Yinan Wang ${ }^{1}$ iD
}

Accepted: 22 March 2021 / Published online: 27 March 2021

(c) The Author(s), under exclusive licence to Springer Nature B.V. 2021, corrected publication 2021

\begin{abstract}
The coronavirus disease 2019 (COVID-19) pandemic threatens human beings' livelihoods and mental health, which lowers their well-being and gives rise to anxiety. This study examines whether there is a causal relationship (and, if so, in which direction) between people's well-being and COVID-19 anxiety. Two hundred and twenty-two participants ( $54.50 \%$ female, $M_{\mathrm{age}}=31.53, S D=8.17$ ) from 26 provinces of China completed measures of subjective well-being (SWB) and COVID-19 anxiety at three key nodes of the development of COVID-19 in China. The results showed that people's SWB and COVID-19 anxiety fluctuated with the peak (T1), decline (T2), and trough stages (T3) of the COVID19 pandemic. Meanwhile, the cross-lagged analysis showed that the participants' SWB at T0 (pre-pandemic stage; the base level of SWB) and T1 could significantly predict their COVID-19 anxiety at T1 and T2 respectively. However, SWB at T2 was not associated with the COVID-19 anxiety at T3. Furthermore, COVID-19 anxiety could not predict subsequent SWB from T1 to T3. The current findings contribute to clarifying the causal relationship between well-being and anxiety through the development of epidemics, as well as finding ways to alleviate people's COVID-19 anxiety.
\end{abstract}

Keywords COVID-19 anxiety $\cdot$ Subjective well-being $\cdot$ Longitudinal design $\cdot$ Cross-lagged analysis · China

\section{Introduction}

In December 2019, coronavirus disease 2019 (COVID-19) emerged in Wuhan, China. Two months later, the epidemic had spread across China and reached its peak level $(15,153$ daily cases, 254 daily deaths; Chinese Center for Disease Control and Prevention, 2020, February 15th). Because of the policies adopted by the Chinese government, by the end of April, the number of confirmed cases in China had declined sharply and remained below

Yinan Wang

yynnwang@gmail.com

1 Beijing Key Laboratory of Applied Experimental Psychology, National Demonstration Center for Experimental Psychology Education (Beijing Normal University), Faculty of Psychology, Beijing Normal University, Beijing, China 
1000. Globally, by the end of April 2020, the virus has spread to more than 210 countries and led to more than 200,000 deaths (World Health Organization, 2020).

As a massive global crisis, the COVID-19 pandemic threatens people's mental health and gives rise to mental illness (Ettman et al., 2020; Fabian et al., 2020; Kachanoff et al., 2020; Qiu et al., 2020; Rosen et al., 2020; Tull et al., 2020; Xu et al., 2020). Previous cross-sectional studies showed that mental health (i.e., well-being) and mental illness (i.e., anxiety) were related but distinct phenomena (Keyes, 2005; Keyes et al., 2002). However, it remains unknown whether mental health would lower mental illness, or vice versa, in unprecedented epidemics. To determine this, the current study examined the relationship between individuals' subjective well-being (SWB) and COVID-19 anxiety using three waves data from the peak, decline, and trough stages of the COVID-19 pandemic in China.

\subsection{COVID-19 Pandemic Increases Anxiety and Lowers SWB}

Accumulating evidence shows that the COVID-19 epidemic has caused unbearable anxiety in people around the world (Rosen et al., 2020; Xiang et al., 2020). COVID-19 anxiety refers to an emotional state of nervousness experienced by individuals caused by the COVID-19 epidemic and fluctuated as the epidemic developed. In China, COVID-19 anxiety had a great impact on people's mental health and quality of life (Cao et al., 2020; Qiu et al., 2020). In the U.S., mean levels of anxiety were found to be heightened and sustained during mid-March 2020 (Rosen et al., 2020). Furthermore, a study in Spain also reported an increased prevalence of COVID-19 anxiety in most of the respondents (OdriozolaGonzález et al., 2020).

The COVID-19 pandemic has not only increased people's anxiety but also reduced their SWB (Kachanoff et al., 2020; Qiu et al., 2020; Rosen et al., 2020; Xu et al., 2020). SWB represents people's appraisals and evaluations of their own lives (Diener et al., 2018). It consists of both cognitive (e.g., life satisfaction) and emotional components (e.g., positive and negative emotions; Diener et al., 1999), and it has been demonstrated to be a general psychological factor embodying people's physical health, psychological health, and social adaption. In the last 4 decades, SWB has been demonstrated to be a general psychological factor embodying people's physical health, psychological health, social adaption and even academic or work achievements (Diener et al., 2018). Therefore, more research is needed to examine why the COVID-19 pandemic has led to decrease in SWB.

One recent study suggests that the threat of COVID-19 predicts both increased distress and decreased SWB (Kachanoff et al., 2020). However, considering the cross-sectional nature of previous studies, it remains unknown whether there is a causal relationship (and, if so, in which direction) between people's SWB and COVID-19 anxiety.

\subsection{Relationship between SWB and COVID-19 Anxiety}

Existing evidence shows that SWB is negatively related to anxiety (Grieve et al., 2013; Lepp et al., 2014). To understand the relationship between SWB and people's evaluation of life events, Diener (1984) proposed bottom-up and top-down theories. From the perspective of the bottom-up theory, the positive emotion of past pleasant experiences contributes to increasing SWB, whereas the negative emotion of unpleasant experiences might decrease one's current evaluation of the whole life (Stallings et al., 1997). Hence, the emergence of COVID-19 might first have made people feel anxious about their health and safety, which might then have lowered their SWB. 
In contrast, the top-down theory of well-being indicates that the reverse may be correct. SWB has a strong effect on several life domains, and higher SWB predicts positive emotional reflection and evaluation towards a certain life event (Diener, 1984). Based on the top-down theory, some psychologists elucidate the role of SWB as a psychological resource in coping with stress and challenges (Fredrickson, 2004; Huppert \& Baylis, 2004). They indicate that high SWB may enhance people's resilience against stressors in negative life events. Efficient well-being therapy in anxiety interventions proffers some evidence supporting these theories that well-being may prevent people from becoming anxious. For example, intervention research shows that patients who receive well-being therapy are encouraged to self-observe to increase their SWB, which can result in lower anxiety levels in future assessments (Fava et al., 2017). Therefore, according to the topdown theory, people with lower SWB may hold a more pessimistic view about the development of the epidemic, leading to heightened levels of anxiety. Furthermore, with respect to the potential protective role of SWB in this theory, an individual's SWB level before the epidemic may also have a significant influence on their COVID-19 anxiety.

Although intervention research supports the top-down theory, it remains unclear whether the top-down theory can be applied to the interpretation of the relationship between SWB and anxiety in natural settings, especially during massive epidemics. To determine this, the current study aimed to examine the relationship between individuals' SWB and COVID-19 anxiety using longitudinal data throughout the development of the COVID-19 pandemic.

There are three major contributions of the present study as follows. First, this study might contribute to discriminating the relation of the top-down and bottom-up theories of SWB. As two competing models, the top-down and bottom-up theories have generated contradictory findings. The current study helps determine which one would provide an appropriate explanation for the relationship between SWB and COVID-19 anxiety at different stages of the COVID-19 pandemic. Second, to our knowledge, existing studies that examine or integrate the top-down and bottom-up theories usually adopt natural and longitudinal research using long time intervals, such as years (Brief et al., 1993; Feist et al., 1995; Nakazato et al., 2011). To extend existing research, the present study collected three waves of data within a short time interval of a month, which corresponds to the inflection point of the COVID-19 epidemic, similar to a field experiment. In this way, we might not only capture the subtle fluctuation between SWB and anxiety but also examine the topdown and bottom-up theories from a dynamic perspective. Third, in practice, the current study sheds light on finding feasible ways to alleviate people's COVID-19 anxiety from the perspective of positive psychology.

\subsection{Overview of the Current Study}

To unfold the relationship between people's SWB and their COVID-19 anxiety, we collected three waves data on SWB and COVID-19 anxiety at three key stages of the development of COVID-19 in China: the peak (T1; February 13th, 2020), the decline (T2; March 13th, 2020), and the trough (T3; April 8th, 2020) stages. In fact, the three waves of data belong to a longitudinal project concerning the relationship between SWB and anxiety. Thus, we measured the participants' trait anxiety and SWB on January 3rd of 2020 (T0) when COVID-19 was not yet declared an emergent public health event. Given that people knew little about the infectivity and harmfulness of COVID-19 and it had not led to 
widespread panic and global crises on January 3rd (Zhao et al., 2020), we described T0 as "before the pandemic".

Using data from before the pandemic and three subsequent waves of data from throughout the peak and the trough of the COVID-19 pandemic, the aims of our study were to examine (1) whether people's SWB and anxiety fluctuated dynamically with the development of the COVID-19 pandemic; (2) whether there was a causal relationship between SWB and COVID-19 anxiety; and (3) if so, did people's SWB alleviate their COVID-19 anxiety or did their COVID-19 anxiety damage their SWB?

\section{Methods}

\subsection{Participants and Procedure}

The current sample size was determined by the availability of potential participants over a four-month period. The participants were 222 Chinese adults (54.50\% female, $M_{\text {age }}=31.53, S D=8.17$ ) from 26 provinces via a survey website (www.wjx.cn). We collected our baseline data on January 3rd (T0). Then, we followed-up the participants on February 13th (T1; the peak stage of the COVID-19 pandemic in China; more than 3000 newly confirmed cases in China per day), March 13th (T2; the decline stage of COVID19 in China; approximately 30 newly confirmed indigenous cases in China per day), and April 8th (T3; the trough stage, a period when the COVID-19 was generally under control in China; lower than 10 newly confirmed cases in China per day). Of all the participants, $164(73.87 \%), 123(54.41 \%)$, and $161(72.52 \%)$ participated in the online surveys at T1, T2, and T3, respectively. We obtained participants' informed consent in accordance with the guidelines of the ethics board of the Faculty of Psychology, Beijing Normal University.

We conducted Little's (1988) missing completely at random (MCAR) test for missing data. The Little's MCAR test of all the variables was significant, $\chi^{2}(37)=73.37, p<0.001$, illustrating the hypothesis that the data is MCAR could be rejected. Thus, we used full information maximum likelihood estimation (FIML), a reliable method to deal with nonMCAR missing values, in our study (Enders \& Bandalos, 2001). Participants with complete versus incomplete data significantly differed in their gender, $\chi^{2}(1)=5.48, p=0.02$; age, $t(220)=4.17, p<0.001$; trait anxiety at T0, $\chi^{2}(41)=59.62, p=0.03$, and SWB at T0, $t(220)=3.79, p<0.001$; but did not differ in their income at T0, $\chi^{2}(7)=10.62, p=0.937$ (see Table S1). All the 222 participants were included in the analyses.

\subsection{Measures}

\subsubsection{COVID-19 Anxiety at T1, T2, and T3}

COVID-19 anxiety was assessed using the 10-item Self-check and Self-inspect Scale for COVID-19 Anxiety (Chinese Psychological Society, 2020, February 9, see Table S2). The participants were asked about their anxious behaviours and mood after the outbreak of COVID-19 in the past month (e.g., "I feel more irritable, dissatisfied or angry than usual."). Items were rated on a 5-point scale from $1=$ almost never to $5=$ almost always. We computed the total scores by adding all the items, with higher scores representing higher levels 
of COVID-19 anxiety. The Cronbach's alpha values were $0.87,0.86$, and 0.87 at T1, T2, and $\mathrm{T} 3$, respectively.

\subsubsection{SWB at T0, T1, T2, and T3}

SWB was assessed using the 18-item well-validated Chinese version of the General Well-Being Schedule (GWB; Duan, 1996; Fang et al., 2018; Fazio, 1977; Li et al., 2015, see Table S3). The GWB includes six dimensions: anxiety, depression, general health, positive well-being, self-control, and vitality (Brook et al., 1979). The participants reported their levels of SWB in the past month (e.g., "How happy, satisfied, or pleased have you been with your personal life?"). Total scores were computed and used in analyses, with higher scores indicating higher levels of SWB. The Cronbach's alpha values were $0.88,0.91$, and 0.91 at $\mathrm{T} 1, \mathrm{~T} 2$, and $\mathrm{T} 3$, respectively.

\subsubsection{Trait Anxiety at T0}

Trait anxiety was assessed using the 20-item Chinese version of the Trait Anxiety Subscale of the State-Trait Anxiety Inventory (STAI; Chen et al., 2014; Li \& Qian, 1995; Spielberger et al., 1983, see Table S4). The participants rated their levels of general anxiety (e.g., "I wish I could be as happy as others seem.") on a 4-point scale from $1=$ not at all to $4=$ very much so. Higher scores indicated higher levels of trait anxiety. In the current study, the Cronbach's alpha was 0.89 at T0.

\subsubsection{Demographic Variables at T0}

Participants reported their age, gender $(1=$ male, $2=$ female $)$, and monthly income (from 1= "lower than 5,000 yuan" to 7="higher than 35,000 yuan"; the mode of monthly income is "between 5000 and 10,000 yuan", 97 adults, 43.69\%) at T0. The aforementioned variables were considered as covariates in the model due to their significant correlations with anxiety and SWB in previous studies (e.g., Lofors et al., 2006; Merikangas et al., 2003; Steptoe et al., 2015).

\subsection{Planned Analysis}

First, to investigate whether SWB and COVID-19 anxiety changed during this period, we conducted mixed-effects modeling using the nlme package in R (Pinheiro et al., 2014). Compared to conventional repeated-measures ANOVA which involves only fixed effects, mixed-effects modeling considers both fixed effects and random effects, which is preferred for analyzing non-random data and data with a large number of missing values. When considering the subject as one random effect, it could prevent the following three problems that might give us problematic results: (1) data with a relatively high rate of nonrandom missing responses, (2) high variability among participants, and (3) the fact that the response of the participants might depend on their past making the data nonindependent (Baayen et al., 2008; Bagiella et al., 2000; Krueger \& Tian, 2004). Then, we used the multcomp package in R (Bretz et al., 2016) to perform the Bonferroni-corrected post-hoc test. 
Second, to examine whether the base level of SWB would influence the trajectories of SWB and COVID-19 anxiety, we divided the subjects into two groups based on SWB assessed at T0 (high-SWB group and low-SWB group), which could be considered the base level of SWB. We fit two linear mixed-effects models with SWB and COVID-19 anxiety as the outcome variables, with fixed effects of time points (T1, T2, and T3) and SWB-level groups (both of them were considered dummy variables) and their interaction, to investigate whether the base level of SWB could impact the trajectory of SWB and COVID-19 anxiety from T1 to T3. Post-hoc tests were also conducted to better interpret the impact.

Third, to explore the relationships between SWB and COVID-19 anxiety, three-wave cross-lagged panel models (CLPMs) were tested using structural equation modeling (SEM) in the lavaan package in $\mathrm{R}$ (Rosseel, 2012). Compared with cross-sectional data collected at a specific point in time that could only help to conjecture causality relying on theoretical inference, panel data with repeated measures of variables at multiple points in time could help to describe the directional influences that one variable has on another across different time points (Falkenström et al., 2020; Shingles, 2007).

The cross-lagged panel model would contain both autoregressive paths and cross-lagged paths. Specifically, the autoregressive paths could suggest the degree of how the current state of a variable would depend on its past, reflecting the persistence in the variable over time. Cross-lagged terms are used to reflect causality, reflecting whether the perturbation of one variable could predict the perturbation of another variable, and estimating the reciprocal relationships between variables over time. To investigate the cross-lagged effects, four competing models were examined: (1) a model with only autoregressive paths that could suggest the stability of the construct, (2) a model with autoregressive paths and crosslagged paths from SWB to subsequent COVID-19 anxiety, (3) a model with autoregressive paths and cross-lagged paths from COVID-19 anxiety to subsequent SWB, and (4) a fully cross-lagged model with both autoregressive paths and cross-lagged paths of SWB and COVID-19 anxiety. Considering the potential correlation with SWB and COVID-19 anxiety, sex, age, income, and trait anxiety were considered covariates in the model (Jebb et al., 2018; Mirowsky \& Schieman, 2008; Moksnes \& Espnes, 2013; Thomsen et al., 2016).

The models' goodness of fit was evaluated using fit indexes including the $\chi^{2}$ test (acceptable when $\chi^{2} / d f$ ratio $\leq 3$ and good when $\chi^{2} / d f$ ratio $\leq 2$ ), comparative fit index (CFI; acceptable when $\mathrm{CFI} \geq 0.95$ and good when $\mathrm{CFI} \geq 0.97$ ), root-mean-square error of approximation (RMSEA; acceptable when RMSEA $\leq 0.08$ and good when RMSEA $\leq 0.05$ ), and standardized root mean squared residual (SRMR; acceptable when SRMR $\leq 0.10$ and good when SRMR $\leq 0.05$; Schermelleh-Engel et al., 2003).

\subsection{Results}

\subsubsection{Descriptive Statistics}

Table 1 showed the descriptive statistics for all the variables in the present study. SWB and COVID-19 anxiety negatively correlated across all time points. In addition, trait anxiety was highly correlated with SWB and COVID-19 anxiety measured at every time point.

The trajectories of SWB and COVID-19 anxiety were presented in Fig. 1. For SWB, compared to the fixed effects model, the mixed effects model used for repeated-measure ANOVA could offer better fit to the data, $\chi_{\text {diff }}^{2}(1)=297.21, p<0.001$ (see Table S5). In 


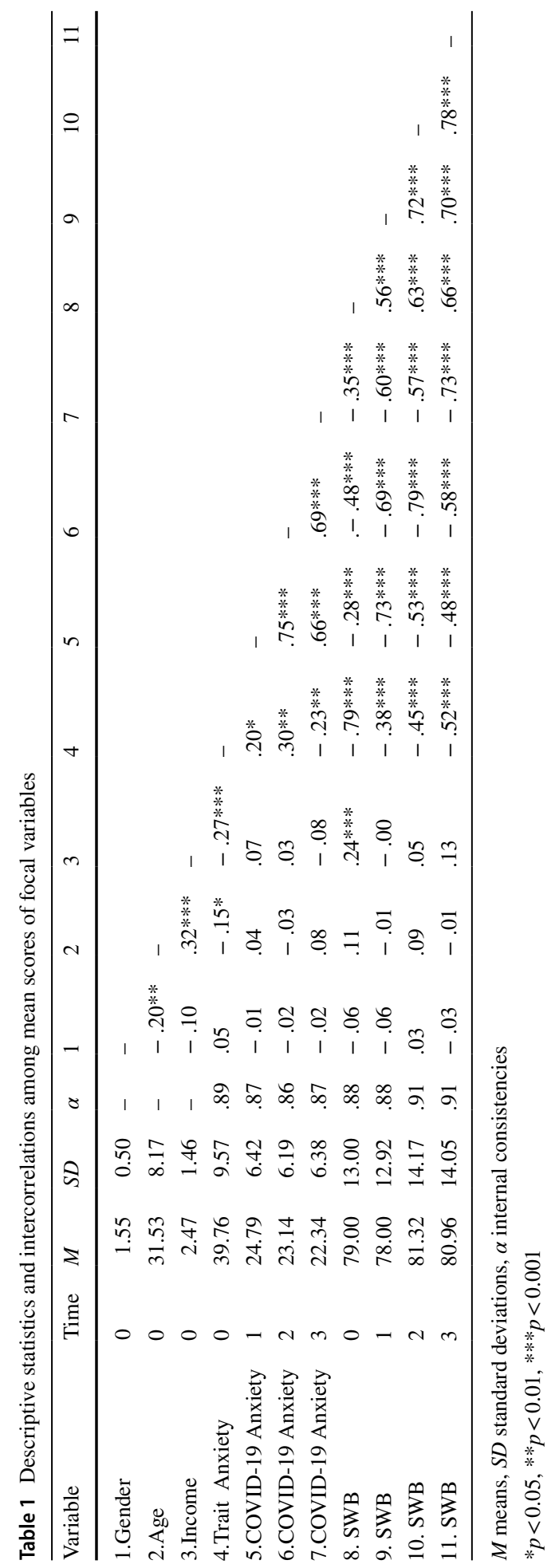




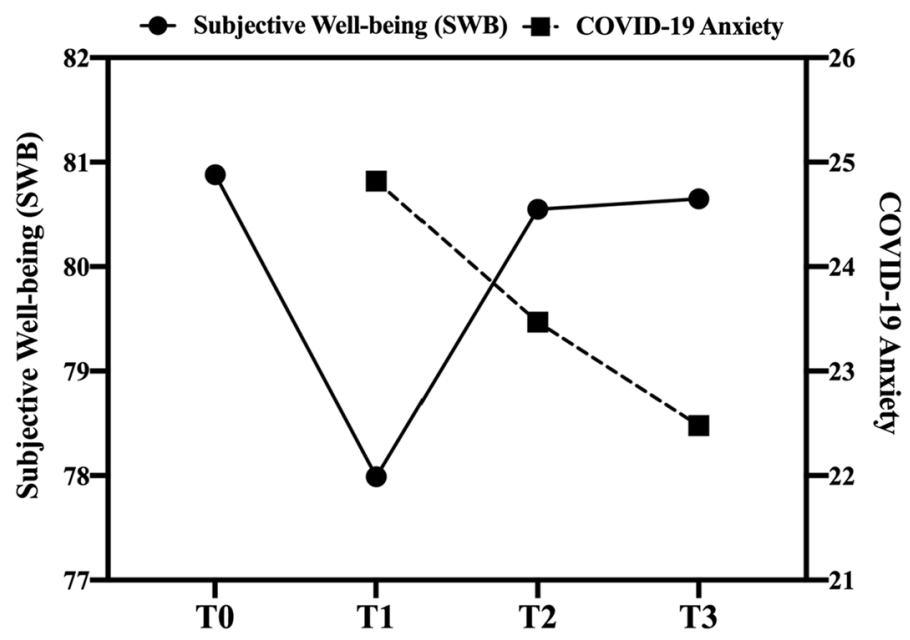

Fig. 1 The trajectories of SWB and COVID-19 anxiety from T0 to T3. T0 = pre-COVID stage, T1=peak stage of COVID-19 in China, T2= decline stage of COVID-19 in China, T3= trough stage of COVID-19 in China

the mixed effect model, after disaggregating random effects, the main effect of time points was significant, $F_{(3,445)}=4.754, p=0.003$. As the results of post-hoc tests suggested (see Table S6) there was an obvious drop of SWB from T0 (i.e., the pre-pandemic stage) to T1 (i.e., the peak stage; $M_{\mathrm{WB} 1-0}=-2.89, p=0.005$ ). From T1 to T2 (i.e., the decline stage), SWB began to rebound $\left(M_{\mathrm{WB} 2-1}=2.56, p=0.049\right)$, and then kept stable from T2 to T3 (i.e., the trough stage; $\left.M_{\mathrm{WB} 3-2}=0.10, p=1.000\right)$.

For COVID-19 anxiety, following the same testing process, the mixed effect model was also better fitting than fixed effect one, $\chi_{\text {diff }}^{2}(1)=196.42, p<0.001$ (see Table S7). Levels of COVID-19 anxiety were different among three time points $\left(F_{(3,268)}=16.95, p<0.001\right)$. Post-hoc tests (see Table S8) indicated that COVID-19 anxiety declined from T1 (i.e., the peak stage) to T2 (i.e., the decline stage; $M_{\mathrm{COV} 2-1}=-1.35, p=0.006$ ), and continued marginally declining from T2 to T3 $\left(M_{\mathrm{COV} 3-2}=-0.99, p=0.078\right)$.

\subsubsection{The impact of the Base Level of SWB (T0) on the Trajectories of SWB and COVID-19 Anxiety from T1 to T3}

For the trajectory of COVID-19 anxiety from T1 to T3, compared to the model with only fixed effects, the mixed effects model could fit the data better $\left(\chi^{2}(1)=188.96, p<0.001\right)$ as the variance in intercepts among participants $(S D=5.01,95 \%$ CI $[4.41,5.68])$ accounted for $68 \%$ of the variance of the model. The results of the mixed effects model could be seen in Table 2. The results of post-hoc test with Bonferroni corrections suggested the different levels and trajectories of COVID-19 anxiety between two groups with different base levels of SWB (see Table 3). There was no difference of COVID-19 anxiety levels at T1 between two groups $\left(M_{\mathrm{T} 1 \text { (high-low) }}=-2.56, p=0.102\right)$, while participants with low base level of SWB would show higher levels of COVID-19 anxiety at T2 and T3 compared with those with high base level of SWB $\left(M_{\mathrm{T} 2 \text { (high-low) }}=-5.20, p<0.001 ; M_{\mathrm{T} 3 \text { (high-low) }}=-3.45\right.$, $p=0.005)$. As for the trajectory, for participants with low base level of SWB, their COVID-19 anxiety would keep stable from T1 to T2 $\left(M_{\mathrm{COV} 2-1}=0.24, p=1.000\right)$, and then 
Table 2 The impacts of baseline SWB on the trajectories of COVID-19 Anxiety from T1 to T3

\begin{tabular}{|c|c|c|c|c|c|c|}
\hline \multirow[t]{2}{*}{ Predictors } & \multicolumn{3}{|c|}{ Mixed effects model } & \multicolumn{3}{|c|}{ Fixed effects model } \\
\hline & Estimates & CI & $p$ & Estimates & $\mathrm{CI}$ & $p$ \\
\hline (Intercept) & 26.20 & {$[24.83,27.57]$} & $<0.001$ & 26.08 & {$[24.67,27.49]$} & $<0.001$ \\
\hline $\mathrm{T} 2^{\mathrm{a}}$ & 0.24 & {$[-1.06,1.54]$} & 0.720 & 0.04 & {$[-2.17,2.25]$} & 0.973 \\
\hline $\mathrm{T}^{\mathrm{a}}$ & -1.82 & {$[-3.01,-0.62]$} & 0.003 & -1.94 & {$[-3.95,0.07]$} & 0.059 \\
\hline High-SWB ${ }^{b}$ & -2.56 & {$[-4.40,-0.72]$} & 0.006 & -2.33 & {$[-4.22,-0.45]$} & 0.016 \\
\hline T2 $\times$ High $-S W B$ & -2.64 & {$[-4.34,-0.94]$} & 0.002 & -2.69 & {$[-5.59,0.22]$} & 0.070 \\
\hline T3 $\times$ High $-S W B$ & -0.89 & {$[-2.47,0.68]$} & 0.266 & -0.86 & {$[-3.55,1.82]$} & 0.528 \\
\hline \multicolumn{7}{|l|}{ Random effects } \\
\hline$\sigma^{2}$ & \multicolumn{3}{|l|}{12.03} & \multicolumn{3}{|l|}{-} \\
\hline$\tau_{00}$ & \multicolumn{3}{|l|}{$25.07_{\text {subject }}$} & \multicolumn{3}{|l|}{-} \\
\hline $\mathrm{ICC}$ & \multicolumn{3}{|l|}{0.68} & \multicolumn{3}{|l|}{-} \\
\hline $\mathrm{N}$ & \multicolumn{3}{|l|}{$178_{\text {subject }}$} & \multicolumn{3}{|l|}{-} \\
\hline \multicolumn{7}{|c|}{ Model comparison (mixed effects model vs. fixed effects model) } \\
\hline$\chi^{2}$ & \multicolumn{6}{|c|}{188.96} \\
\hline$d f$ & \multicolumn{6}{|l|}{1} \\
\hline$p$ & \multicolumn{6}{|l|}{$<0.001$} \\
\hline
\end{tabular}

The significance level was in bold when $p<0.05, p<0.01$, or $p<0.001$

$\sigma^{2}=$ fixed effects variance; $\tau_{00}=$ random intercept variance; ICC indicates how much variance is explained by a random effect

"We dummy coded the "time" variable, with the T0 as the reference

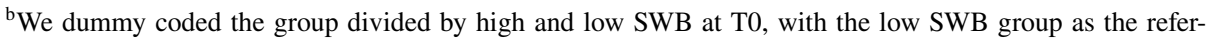
ence group.

Table 3 Bonferroni comparison for COVID-19 anxiety between groups with different base levels of SWB (mixed effects model)

\begin{tabular}{|c|c|c|c|c|c|c|}
\hline & $\begin{array}{l}\text { COVID-19 } \\
\text { Anxiety (I) }\end{array}$ & $\begin{array}{l}\text { COVID-19 } \\
\text { Anxiety (J) }\end{array}$ & Estimates (J-I) & $S E$ & $t$ & $p$ \\
\hline \multirow[t]{3}{*}{ G1 } & \multirow[t]{2}{*}{$\mathrm{T} 1$} & $\mathrm{~T} 2$ & 0.24 & 0.67 & 0.36 & 1.000 \\
\hline & & T3 & -1.82 & 0.61 & -2.98 & 0.047 \\
\hline & $\mathrm{T} 2$ & T3 & -2.06 & 0.68 & -3.03 & 0.041 \\
\hline \multirow[t]{3}{*}{ G2 } & \multirow[t]{2}{*}{$\mathrm{T} 1$} & $\mathrm{~T} 2$ & -2.40 & 0.56 & -4.31 & $<0.001$ \\
\hline & & $\mathrm{T} 3$ & -2.71 & 0.52 & -5.19 & $<0.001$ \\
\hline & $\mathrm{T} 2$ & T3 & -0.31 & 0.56 & -0.55 & 1.000 \\
\hline \multirow[t]{3}{*}{ G1 versus G2 } & $\mathrm{T} 1(\mathrm{G} 1)$ & $\mathrm{T} 1(\mathrm{G} 2)$ & -2.56 & 0.94 & -2.73 & 0.102 \\
\hline & $\mathrm{T} 2(\mathrm{G} 1)$ & $\mathrm{T} 2(\mathrm{G} 2)$ & -5.20 & 1.02 & -5.10 & $<0.001$ \\
\hline & T3(G1) & T3(G2) & -3.45 & 0.94 & -3.66 & 0.005 \\
\hline
\end{tabular}

The significance level was in bold when $p<0.05, p<0.01$, or $p<0.001$

$\mathrm{G} 1=$ group with the low base level of SWB, G2= group with the high base level of SWB.

dropped significantly from $\mathrm{T} 2$ to $\mathrm{T} 3\left(M_{\mathrm{COV} 3-2}=-2.06, p=0.041\right)$. However, for those with high level of SWB, the level of COVID-19 anxiety would significantly decrease from 
Fig. 2 The trajectory of COVID19 anxiety from $\mathrm{T} 1$ to $\mathrm{T} 3$ in two groups with different SWB baseline level (T0)

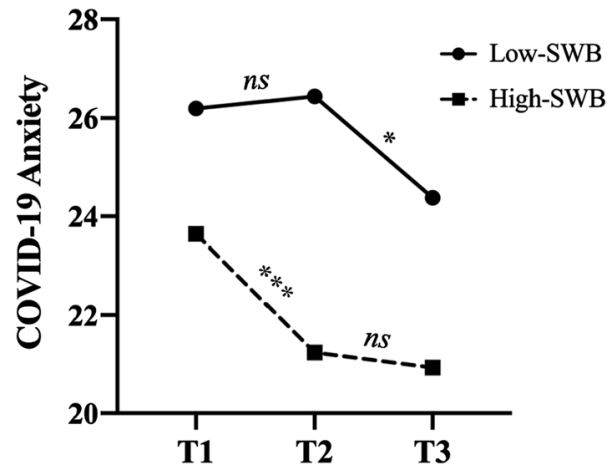

Table 4 The impacts of baseline SWB on the trajectories of SWB from T1 to T3

\begin{tabular}{|c|c|c|c|c|c|c|}
\hline \multirow[t]{2}{*}{ Predictors } & \multicolumn{3}{|c|}{ Mixed effects model } & \multicolumn{3}{|c|}{ Fixed effects model } \\
\hline & Estimates & CI & $p$ & Estimates & $\mathrm{CI}$ & $p$ \\
\hline (Intercept) & 72.78 & {$[70.05,75.50]$} & $<0.001$ & 73.07 & [70.28. 75.85] & $<0.001$ \\
\hline $\mathrm{T} 2$ & 0.57 & {$[-2.17,3.31]$} & 0.684 & 0.53 & {$[-3.83,4.90]$} & 0.811 \\
\hline T3 & 1.36 & {$[-1.15,3.87]$} & 0.289 & 1.29 & {$[-2.69,5.27]$} & 0.525 \\
\hline High-SWB & 11.51 & {$[7.83,15.19]$} & $<0.001$ & 11.09 & {$[7.35,14.82]$} & $<0.001$ \\
\hline T2 $\times$ High $-S W B$ & 3.45 & {$[-0.13,7.03]$} & 0.059 & 3.99 & {$[-1.76,9.73]$} & 0.174 \\
\hline T3×High-SWB & 2.29 & {$[-1.02,5.60]$} & 0.176 & 2.56 & {$[-2.76,7.87]$} & 0.345 \\
\hline \multicolumn{7}{|l|}{ Random effects } \\
\hline$\sigma^{2}$ & 53.57 & & & - & & \\
\hline$\tau_{00}$ & $94.14_{\text {subject }}$ & & & - & & \\
\hline ICC & 0.64 & & & - & & \\
\hline $\mathrm{N}$ & $178_{\text {subject }}$ & & & - & & \\
\hline \multicolumn{7}{|l|}{ Model comparison } \\
\hline$\chi^{2}$ & 155.71 & & & & & \\
\hline$d f$ & 1 & & & & & \\
\hline$p$ & $<0.001$ & & & & & \\
\hline
\end{tabular}

The significance level was in bold when $p<0.05, p<0.01$, or $p<0.001$

$\sigma^{2}=$ fixed effects variance; $\tau_{00}=$ random intercept variance; ICC indicates how much variance is explained by a random effect.

T1 to T2 $\left(M_{\mathrm{COV} 2-1}=-2.40, p<0.001\right)$, while keep relatively consistent from $\mathrm{T} 2$ to $\mathrm{T} 3$ $\left(M_{\mathrm{COV} 3-2}=-0.31, p=1.000\right.$; see Fig. 2$)$.

For the trajectory of SWB from T1 to T3, compared to the model with only fixed effects, the mixed effects model shows better goodness of fit $\left(\chi^{2}(1)=155.71, p<0.001\right)$ as the variance in intercepts among participants $(S D=9.70,95 \%$ CI $[8.49,11.08])$ accounted for $64 \%$ of the variance of the model. The results of the mixed effects model is shown in Table 4. The results of post-hoc test with Bonferroni corrections suggested the different levels and trajectories of SWB from T1 to T3 between two groups with different base levels of SWB assessed at T0 (see Table 5).

For participants with low base level of SWB at T0, SWB levels at consequent three time points were significantly lower compared with those with high base level of SWB 
Table 5 Bonferroni comparison for mixed effects model of SWB between groups with different base levels of SWB (mixed effects model)

\begin{tabular}{lllclrr}
\hline & SWB (I) & SWB (J) & Estimates (J-I) & \multicolumn{1}{c}{$S E$} & $t$ & \multicolumn{2}{c}{$p$} \\
\hline G1 & T1 & T2 & 0.57 & 1.40 & 0.41 & 1.000 \\
& & T3 & 1.36 & 1.28 & 1.06 & 1.000 \\
G2 & T2 & T3 & 0.79 & 1.43 & 0.55 & 1.000 \\
& T1 & T2 & 4.02 & 1.18 & 3.42 & $\mathbf{0 . 0 1 1}$ \\
& & T3 & 3.65 & 1.10 & 3.31 & $\mathbf{0 . 0 1 6}$ \\
G1 versus G2 & T2 & T3 & -0.37 & 1.19 & -0.31 & 1.000 \\
& T1(G1) & T1(G2) & 11.51 & 1.88 & 6.13 & $<\mathbf{0 . 0 0 1}$ \\
& T2(G1) & T2(G2) & 14.96 & 2.05 & 7.29 & $<\mathbf{0 . 0 0 1}$ \\
& T3(G1) & T3(G2) & 13.80 & 1.89 & 7.31 & $<\mathbf{0 . 0 0 1}$ \\
\hline
\end{tabular}

The significance level was in bold when $p<0.05$ or $p<0.001$

$\mathrm{G} 1=$ group with the low base level of SWB, G2 = group with the high base level of SWB.

Fig. 3 The trajectory of SWB from T1 to T3 in two groups with different SWB baseline level (T0)

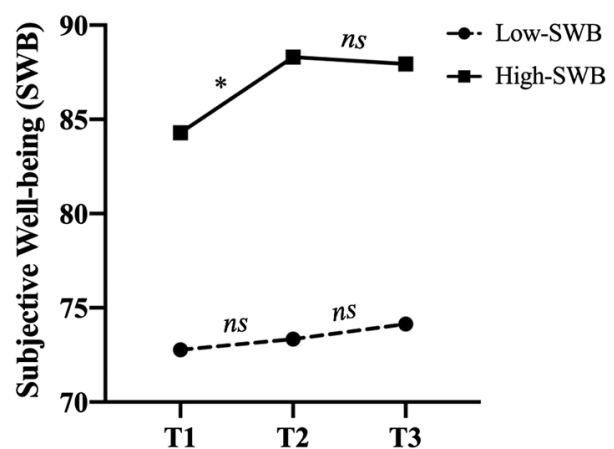

$\left(M_{\mathrm{T} 1 \text { (high-low) }}=11.51, \quad p<0.001 ; \quad M_{\mathrm{T} 2 \text { (high-low) }}=14.96, \quad p<0.001 ; \quad M_{\mathrm{T} 3 \text { (high-low) }}=13.80\right.$, $p<0.001)$. As for the trajectory, for participants with low base level of SWB, their SWB level kept stable from T0 to T3 $\left(M_{\mathrm{WB} 2-1}=0.57, p=1.000 ; M_{\mathrm{WB} 3-2}=0.79, p=1.000\right)$, while for those with high base level of SWB, the SWB level significantly increased from T1 to T2 $\left(M_{\mathrm{WB} 2-1}=4.02, p=0.011\right)$ and kept relatively stable from $\mathrm{T} 2$ to $\mathrm{T} 3$ $\left(M_{\mathrm{WB} 3-2}=-0.37, p=1.000\right.$; see Fig. 3$)$.

\subsubsection{Cross-lagged Panel Models}

Four models were tested from the model with only autoregressive paths to the full crosslagged panel model as suggested above. Model fit indices of each model and the results of model comparisons were reported in Table 6 .

Among the four models, autoregressive model (Model 1) did not show an acceptable fit $\left(\chi^{2} / d f=4.61, \mathrm{CFI}=0.974, \mathrm{RMSEA}=0.127, \mathrm{SRMR}=0.053\right)$. Adding the cross-lagged paths from COVID-19 anxiety at preceding time point to subsequent SWB (Model 2) could have better goodness of fit compared to Model $1\left(\chi_{\text {diff }}{ }^{2}(2)=15.07, p<0.001 ; \chi^{2} / d f=3.14\right.$, $\mathrm{CFI}=0.990$, RMSEA $=0.098, \mathrm{SRMR}=0.025$ ). The model with cross-lagged paths from SWB at preceding time point to subsequent COVID-19 anxiety (Model 3) could also fit 


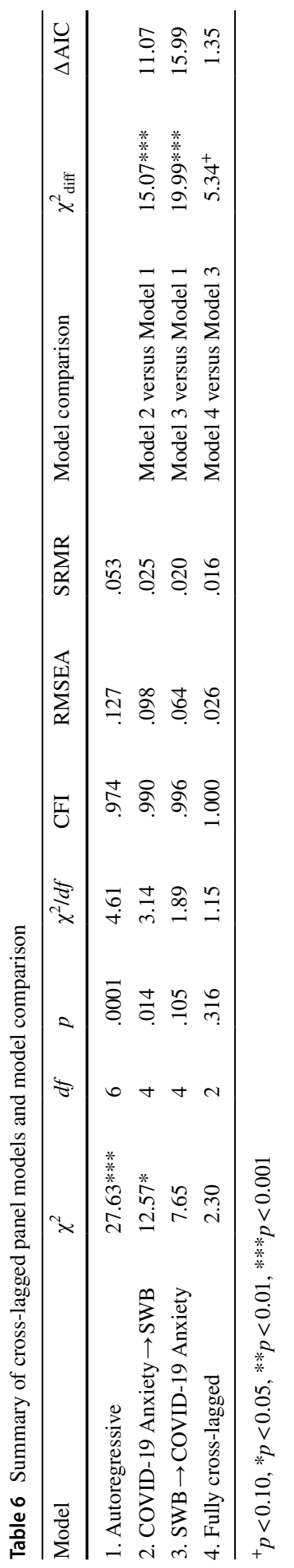


the data better compared to Model $1\left(\chi_{\text {diff }}^{2}(2)=19.99, p<0.001\right)$ and is of acceptable model fit $\left(\chi^{2} / d f=1.89, \mathrm{CFI}=0.996, \mathrm{RMSEA}=0.064, \mathrm{SRMR}=0.020\right)$. And when adding cross-lagged paths from SWB to subsequent COVID-19 anxiety to Model 2, the model (Model 4) could fit the data better $\left(\chi_{\text {diff }}^{2}(2)=10.26, p=0.006 ; \chi^{2} / d f=1.15, \mathrm{CFI}=1.000\right.$, RMSEA $=0.026$, SRMR $=0.016$ ). In general, both Model 3 and 4 have an acceptable fit. While Model 4 can be considered a good fit model regarding all goodness-of-fit indices $\left(\chi_{\text {diff }}^{2}(2)=5.34, p=0.069\right)$. Therefore, the fully cross-lagged model (Model 4$)$ with all autoregressive and cross-lagged paths was the relatively best fitting model.

To confirm the causal link between SWB and COVID-19 anxiety, we added both the autoregressive and cross-lagged path from SWB at T0 to SWB at T1 and COVID-19 anxiety at T1 respectively in Model 4. As shown in Fig. 4, SWB at T0 could negatively predict the level of COVID-19 anxiety at T1 $(B=-0.38, S E=0.11, p=0.001)$, and SWB at T1 predicted lower level of COVID-19 anxiety at T2 $(B=-0.27, S E=0.08, p=0.001)$ after controlling for trait anxiety and demographic variables measured at T0 (i.e., gender, age, and income). However, SWB at T2 was not associated with COVID-19 at T3 ( $B=-0.05$, $S E=0.13, p=0.687)$. Cross-lagged effects from COVID-19 anxiety at preceding time point to subsequent SWB were not significant (T1 to T2: $B=-0.15, S E=0.09, p=0.090$; T2 to T3: $B=0.21, S E=0.11, p=0.065)$.

\section{Discussion}

To understand whether and how people's SWB and COVID-19 anxiety are related to each other, we conducted a three-wave longitudinal study with Chinese participants. There were three main findings in our study. First, consistent with previous research (Kachanoff et al., 2020), COVID-19 pandemic has reduced people's SWB and increased their anxiety. Meanwhile, SWB and anxiety were found to fluctuate dynamically with the development of COVID-19 pandemic. Specifically, the participants' SWB reached its lowest at T1 and recovered gradually at T2 and T3. In contrast, the participants' COVID-19 anxiety reached its peak level at T1 and dropped sharply at T2 and T3. Second, SWB and COVID-19 anxiety were negatively correlated at the three points. Third, the cross-lagged analysis showed

T0

T1

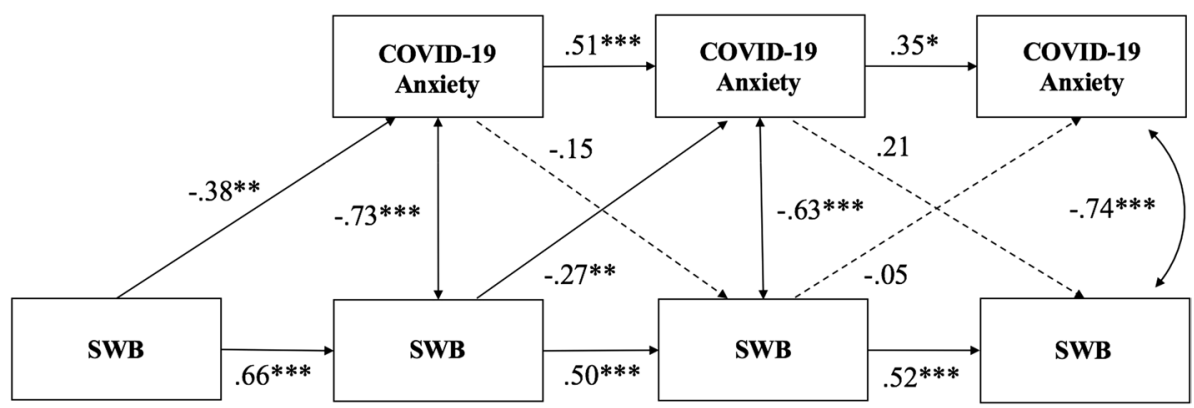

Fig. 4 Fully cross-lagged panel model with SWB at T0. The model considered trait anxiety, gender, age, and income as covariates. $\chi^{2} / d f=2.80, \mathrm{CFI}=0.988$, RMSEA $=0.090$, SRMR $=0.037 .{ }^{*} p<.05,{ }^{*} p<.01$, $* * * p<.001$ 
that SWB at T1 was able to predict COVID-19 anxiety at T2. However, SWB at T2 was not associated with COVID-19 anxiety at T3. Meanwhile, COVID-19 anxiety at the preceding time point could not predict subsequent SWB.

The finding that SWB at T1 predicts COVID-19 anxiety at T2 can be explained by the top-down theory of well-being, which considers SWB as a psychological resource for people to respond effectively to problems and challenges (Fredrickson, 2004; Huppert \& Baylis, 2004). For the first time, the current study extended the top-down theory of well-being to understand the relationship between SWB and anxiety during the development of global epidemic. Individuals with higher SWB have more resilience when confronting negative life events and are better able to adjust their mindset to lower their anxiety during the peak of the COVID-19 pandemic.

More importantly, the current study showed that the causal relationship between SWB and COVID-19 anxiety might change with the development of the COVID-19 pandemic, which might contribute to understanding the boundary condition of the application of the top-down theory of well-being. Different from the tendency that SWB at T1 significantly predicted the subsequent COVID-19 anxiety at T2, SWB at T2 did not predict subsequent COVID-19 anxiety at T3. The results showed that the causal relationship between SWB and COVID-19 anxiety might disappear with the decline of the COVID-19 pandemic.

Moreover, suggested by Diener and his colleagues' seminal reviews (), the confounding variables such as research conditions inevitably involved could account for the ambiguous results of well-being research. For example, as the situation changes, the direction of the causal relationships might become completely inverse and the effect size unstable. Considering the great discrepancy among the pandemic severity of the three time nodes, it is plausible that the perceived pandemic threat might moderate the influence of SWB on COVID-19 anxiety. Although one's SWB level influences their threat assessment and affective reflection to COVID-19, objective pandemic severity also has a significant effect on people's perception. Therefore, it is advisable to remain cautious about giving a determined affirmation of the causal relationship between SWB and pandemic anxiety, and more research is needed to explore the feasible moderated variables. Furthermore, we suggested taking situations into consideration in examining the relationship between SWB and anxiety. In future studies, a contingent top-down theory of well-being might help understand the dynamic relationship between mental health and mental illness.

\section{Implications for Future Research}

The current study found that people's SWB and COVID-19 anxiety fluctuated with the development of the COVID-19 pandemic. Meanwhile, people's SWB at the peak stage of the COVID-19 pandemic predicted their subsequent anxiety. These findings help to clarify the causal relationship from SWB to anxiety and provide a theoretical basis to COVID-19 anxiety intervention.

On the one hand, a high sense of SWB cultivated in individuals' daily lives might serve to buffer the negative effects of unprecedented crises (e.g., COVID-19 pandemic) on mental health. On the other hand, our finding suggests implementing happiness therapy which has already been proved to be efficient in reducing anxiety in patients (Fava et al., 2017), as a practical way to mitigate the mental problems caused by COVID-19. 


\section{Limitations of the Current Research}

The present study has several limitations. First, the sample is relatively small, which might limit the external validity of our findings. Second, the measurement of COVID-19 anxiety was based on subjective self-reports. More objective indicators, such as actual behavior analysis, are preferred in future studies. Third, although the FIML method we used might minimize the impact of missing data to some extent, it might leave unanswered questions regarding individual differences in COVID-19 anxiety. Fourth, considering that SWB might be correlated with financial situation, future studies are suggested to track individuals' income across different stages of the pandemic and examine its impacts on people's mental health during the epidemic.

Supplementary Information The online version contains supplementary material available at https:/doi. org/10.1007/s10902-021-00385-2.

Acknowledgements This work was supported by the National Natural Science Foundation of China (31700978). Zhuojun Wang, Shuyi Luo, and Jianjie Xu are co-first authors and contributed equally to the paper.

\section{References}

Baayen, R. H., Davidson, D. J., \& Bates, D. M. (2008). Mixed-effects modeling with crossed random effects for subjects and items. Journal of Memory and Language, 59, 390-412. https://doi.org/10.1016/j.jml. 2007.12.005.

Bagiella, E., Sloan, R. P., \& Heitjan, D. F. (2000). Mixed-effects models in psychophysiology. Psychophysiology, 37, 13-20. https://doi.org/10.1111/1469-8986.3710013.

Bretz, F., Hothorn, T., \& Westfall, P. (2016). Multiple comparisons using R. . CRC Press.

Brief, A. P., Butcher, A. H., George, J. M., \& Link, K. E. (1993). Integrating bottom-up and top-down theories of subjective well-being: The case of health. Journal of Personality and Social Psychology, 64, 646-653. https://doi.org/10.1037/0022-3514.64.4.646.

Brook, R., Ware, J., Davies-Avery, A., Stewart, A., Donald, C., Rogers, W., Williams, K. N., \& Johnston, S. (1979). Overview of adult health status measures fielded in Rand's health insurance study. Medical Care, 17, 1-131.

Cao, W., Fang, Z., Hou, G., Han, M., Xu, X., Dong, J., \& Zheng, J. (2020). The psychological impact of the COVID-19 epidemic on college students in China. Psychiatry Research, 287, 112934. https://doi.org/ 10.1016/j.psychres.2020.112934.

Chen, X. L., Song, Y. P., \& Sun, H. W. (2014). The relationship between general self-efficacy and state-trait anxiety of adolescents. China Journal of Health Psychology, 22, 418-419. https://doi.org/10.13342/j. cnki.cjhp.2014.03.044.

Chinese Center for Disease Control and Prevention. (2020). Retrieved March 15, 2020 from http://2019n cov.chinacdc.cn/2019-nCoV/index.html.

Chinese Psychological Society. (2020). "Self-check and Self-inspect Scale of COVID-19 Anxiety" on-line, welcome to scan and fill in the answer. Retrieved February 10, 2020 from https://mp.weixin.qq.com/s/ rtlu22EAwkEHd2PKg-pXmg.

Diener, E. (1984). Subjective well-being. Psychological Bulletin, 95, 542-575. https://doi.org/10.1037/ 0033-2909.95.3.542.

Diener, E., Oishi, S., \& Tay, L. (2018). Advances in subjective well-being research. Nature Human Behaviour, 2, 253-260. https://doi.org/10.1038/s41562-018-0307-6.

Diener, E., Suh, E. M., Lucas, R. E., \& Smith, H. L. (1999). Subjective well-being: Three decades of progress. Psychological Bulletin, 125, 276-302. https://doi.org/10.1037/0033-2909.125.2.276.

Duan, J. H. (1996). The trial results and analysis of overall happiness scale in Chinese college students. Chinese Journal of Clinical Psychology, 4, 56-57. https://doi.org/10.16128/j.cnki.1005-3611.1996.01. 016. 
Enders, C. K., \& Bandalos, D. L. (2001). The relative performance of full information maximum likelihood estimation for missing data in structural equation models. Structural Equation Modeling: A Multidisciplinary Journal, 8, 430-457. https://doi.org/10.1207/S15328007SEM0803_5.

Ettman, C. K., Abdalla, S. M., Cohen, G. H., Sampson, L., Vivier, P. M., \& Galea, S. (2020). Prevalence of depression symptoms in US adults before and during the COVID-19 pandemic. JAMA Network Open, 3, e2019686-e2019686. https://doi.org/10.1001/jamanetworkopen.2020.19686.

Fabian, M., Foa, R., \& Gilbert, S. (2020). Wellbeing levels fell during the pandemic but improved under lockdown, data analysis shows. The Conversation. Retrieved December 1, 2020 from https://theco nversation.com/wellbeing-levels-fell-during-the-pandemic-but-improved-under-lockdown-data-analy sis-shows-143367.

Feist, G. J., Bodner, T. E., Jacobs, J. F., Miles, M., \& Tan, V. (1995). Integrating top-down and bottom-up structural models of subjective well-being: A longitudinal investigation. Journal of Personality and Social Psychology, 68, 138-150. https://doi.org/10.1037/0022-3514.68.1.138.

Falkenström, F., Solomonov, N., \& Rubel, J. (2020). Using time-lagged panel data analysis to study mechanisms of change in psychotherapy research: Methodological recommendations. Counselling and Psychotherapy Research, 20, 435-441. https://doi.org/10.1002/capr.12293.

Fang, J. C., Zheng, D., Zhi, X. L., \& Xie, J. (2018). The distribution and correlation of general well-being and personality characteristics of undergraduates in Tianjin. Chinese Journal of Health Psychology, 26, 916-920. https://doi.org/10.13342/j.cnki.cjhp.2018.06.033.

Fava, G. A., Cosci, F., Guidi, J., \& Tomba, E. (2017). Well-being therapy in depression: New insights into the role of psychological well-being in the clinical process. Depression and Anxiety, 34, 801-808. https://doi.org/10.1002/da.22629.

Fazio, A. F. (1977). A concurrent validational study of the NCHS General Well-Being schedule. Vital and health statistics. Series 2, Data evaluation and methods research. https://doi.org/10.1037/e4090 22004-001

Fredrickson, B. L. (2004). The broaden-and-build theory of positive emotions. Philosophical Transactions of the Royal Society of London. Series B: Biological Sciences, 359, 1367-1377. https://doi.org/10. 1098/rstb.2004.1512.

Grieve, R., Indian, M., Witteveen, K., Tolan, G. A., \& Marrington, J. (2013). Face-to-face or Facebook: Can social connectedness be derived online? Computers in Human Behavior, 29, 604-609. https://doi.org/ 10.1016/j.chb.2012.11.017.

Huppert, F. A., \& Baylis, N. (2004). Well-being: Towards an integration of psychology, neurobiology and social science. Philosophical Transactions of the Royal Society of London. Series B: Biological Sciences, 359, 1447-1451. https://doi.org/10.1098/rstb.2004.1520.

Jebb, A. T., Tay, L., Diener, E., \& Oishi, S. (2018). Happiness, income satiation and turning points around the world. Nature Human Behaviour, 2, 33-38. https://doi.org/10.1038/s41562-017-0277-0.

Kachanoff, F., Bigman, Y., Kapsaskis, K., \& Gray, K. (2020). Measuring realistic and symbolic threats of COVID-19 and their unique impacts on wellbeing and adherence to public health behaviors. Retrieved May 1, 2020 from https://psyarxiv.com/5zr3w/.

Keyes, C. L. M. (2005). Mental illness and/or mental health? Investigating axioms of the complete state model of health. Journal of Consulting and Clinical Psychology, 73, 539-548. https://doi.org/10.1037/ 0022-006X.73.3.539.

Keyes, C. L. M., Shmotkin, D., \& Ryff, C. D. (2002). Optimizing well-being: the empirical encounter of two traditions. Journal of Personality and Social Psychology, 82, 1007-1022. https://doi.org/10.1037/ 0022-3514.82.6.1007.

Krueger, C., \& Tian, L. (2004). A comparison of the general linear mixed model and repeated measures ANOVA using a dataset with multiple missing data points. Biological Research for Nursing, 6, 151157. https://doi.org/10.1177/1099800404267682.

Lepp, A., Barkley, J. E., \& Karpinski, A. C. (2014). The relationship between cell phone use, academic performance, anxiety, and Satisfaction with Life in college students. Computers in Human Behavior, 31, 343-350. https://doi.org/10.1016/j.chb.2013.10.049.

Li, W. L., \& Qian, M. Y. (1995). Revision of Chinese college students' norm of state trait anxiety scale. Acta Scientiarum Naturalium Universitatis Pekinensis, 1995, 108-112.

Li, Y., Lan, J., \& Ju, C. (2015). Achievement motivation and attributional style as mediators between perfectionism and subjective well-being in Chinese university students. Personality and Individual Differences, 79, 146-151. https://doi.org/10.1016/j.paid.2015.01.050.

Little, R. J. A. (1988). A test of missing completely at random for multivariate data with missing values. Journal of the American Statistical Association, 83, 1198-1202. https://doi.org/10.1080/01621459. 1988.10478722. 
Lofors, J., Ramírez-León, V., \& Sundquist, K. (2006). Neighbourhood income and anxiety: A study based on random samples of the Swedish population. The European Journal of Public Health, 16, 633-639. https://doi.org/10.1093/eurpub/ck1026.

Merikangas, K. R., Zhang, H., Avenevoli, S., Acharyya, S., Neuenschwander, M., \& Angst, J. (2003). Longitudinal trajectories of depression and anxiety in a prospective community study: The Zurich Cohort Study. Archives of General Ppsychiatry, 60, 993-1000. https://doi.org/10.1001/archpsyc.60.9.993.

Mirowsky, J., \& Schieman, S. (2008). Gender, age, and the trajectories and trends of anxiety and anger. Advances in Life Course Research, 13, 45-73. https://doi.org/10.1016/S1040-2608(08)00003-8.

Moksnes, U. K., \& Espnes, G. A. (2013). Self-esteem and life satisfaction in adolescents-Gender and age as potential moderators. Quality of Life Research, 22, 2921-2928. https://doi.org/10.1007/ s11136-013-0427-4.

Nakazato, N., Schimmack, U., \& Oishi, S. (2011). Effect of changes in living conditions on well-being: A prospective top-down bottom-up model. Social Indicators Research, 100, 115-135. https://doi.org/10. 1007/s11205-010-9607-6.

Odriozola-González, P., Planchuelo-Gómez, Á., Irurtia-Muñiz, M. J., \& de Luis-García, R. (2020). Psychological symptoms of the outbreak of the COVID-19 crisis and confinement in the population of Spain. PsyArXiv. https://doi.org/10.31234/osf.io/mq4fg

Pinheiro, J., Bates, D., Debroy, S., \& Sarkar, D. (2014). R Core Team (2014). nlme: Linear and nonlinear mixed effects models. $R$ Package Version, 3, 117.

Qiu, J., Shen, B., Zhao, M., Wang, Z., Xie, B., \& Xu, Y. (2020). A nationwide survey of psychological distress among Chinese people in the COVID-19 epidemic: Implications and policy recommendations. General Psychiatry, 33, e100213. https://doi.org/10.1136/gpsych-2020-100213.

Rosen, Z., Weinberger-Litman, S. L., Rosenzweig, C., Rosmarin, D. H., Muennig, P., Carmody, E. R., Rao ST, Litman, L. (2020). Anxiety and distress among the first community quarantined in the U.S due to COVID-19: Psychological implications for the unfolding crisis. PsyArXiv. https://doi.org/10.31234/ osf.io/7eq8c

Rosseel, Y. (2012). Lavaan: An R package for structural equation modeling and more. Version 0.5-12 (BETA). Journal of Statistical Software, 48, 1-36.

Schermelleh-Engel, K., Moosbrugger, H., \& Müller, H. (2003). Evaluating the fit of structural equation models: Tests of significance and descriptive goodness-of-fit measures. Methods of Psychological Research Online, 8, 23-74.

Shingles, R. D. (2007). Causal inference in cross-lagged panel analysis. In H. M. Blalock Jr. (Ed.), Causal Models in Panel and Experimental Designs. (1st ed., pp. 219-249). Routledge. https://doi.org/10.4324/ 9781315081670.

Spielberger, C. D., Gorsuch, R. L., Lushene, R., Vagg, P. R., \& Jacobs, G. A. (1983). Manual for the StateTrait Anxiety Inventory (Form Y). . Mind Garden.

Stallings, M. C., Dunham, C. C., Gatz, M., Baker, L. A., \& Bengtson, V. L. (1997). Relationships among life events and psychological well-being: More evidence for a two-factor theory of well-being. Journal of Applied Gerontology, 16, 104-119. https://doi.org/10.1177/073346489701600106.

Steptoe, A., Deaton, A., \& Stone, A. A. (2015). Subjective wellbeing, health, and ageing. The Lancet, 385 , 640-648. https://doi.org/10.1016/S0140-6736(13)61489-0.

Thomsen, D. K., Matthiesen, S., Frederiksen, Y., Ingerslev, H. J., Zachariae, R., \& Mehlsen, M. Y. (2016). Trait anxiety predicts the emotional valence of meaning-making in life stories: A 10-year prospective study. Personality and Individual Differences, 102, 51-55. https://doi.org/10.1016/j.paid.2016.06.059.

Tull, M. T., Edmonds, K. A., Scamaldo, K., Richmond, J. R., Rose, J. P., \& Gratz, K. L. (2020). Psychological outcomes associated with stay-at-home orders and the perceived impact of COVID-19 on daily life. Psychiatry Research, 289, 113098. https://doi.org/10.1016/j.psychres.2020.113098.

World Health Organization. (2020). Retrieved May 1, 2020 from https://covid19.who.int.

Xiang, Y., Yang, Y., Li, W., Zhang, L., Zhang, Q., Cheung, T., \& Ng, C. H. (2020). Timely mental health care for the 2019 novel coronavirus outbreak is urgently needed. The Lancet Psychiatry, 7, 228-229. https://doi.org/10.1016/S2215-0366(20)30046-8.

Xu, J., Ou, J., Luo, S., Wang, Z., Chang, E. C., Claire, N., Novak, C., Shen, J., Zheng, S., \& Wang, Y. (2020). Perceived social support protects lonely people against COVID-19 anxiety: A three-wave longitudinal study in China. Frontiers in Psychology, 11, 2759-2770. https://doi.org/10.3389/fpsyg.2020. 566965.

Zhao, Y., Cheng, S., Yu, X., \& Xu, H. (2020). Chinese public's attention to the COVID-19 epidemic on social media: Observational descriptive study. Journal of Medical Internet Research, 22, e18825. https://doi.org/10.2196/18825. 
Publisher's Note Springer Nature remains neutral with regard to jurisdictional claims in published maps and institutional affiliations. 